\title{
DECOMPOSABLE INVOLUTION CENTRALIZERS INVOLVING EXCEPTIONAL LIE TYPE SIMPLE GROUPS
}

\author{
M. J. CURRAN
}

(Received 11 April 1975; revised 8 October 1975)

\section{Introduction}

There have been investigations (Janko (1966), Janko and Thompson (1966), Yamaki (1972)) of finite groups $G$ which contain a central involution $t$ whose centralizer (in $G$ ) has the form $C(t)=\langle t\rangle \times F$, where $F$ is isomorphic to a non-abelian simple group. Here it is shown such a group cannot be simple when $F$ is isomorphic to an exceptional Lie type simple group of odd characteristic. Specifically the following theorem is proved.

THEOREM 1.1. Let $G$ be a finite group with a central involution $t$ whose centralizer has form

$$
C(t)=\langle t\rangle \times F,
$$

where $F$ is isomorphic to an exceptional Lie type simple group of odd characteristic. Then $G$ has a subgroup of index 2 not containing $t$.

Theorem 1.1 may be combined with the results of Curran to give:

THEOREM 1.2. Let $G$ be a finite group with a central involution $t$ whose centralizer has the structure

$$
C(t)=\langle t\rangle \times F
$$

where $F$ is isomorphic to any alternating simple group or any classical or exceptional Lie type simple group of odd characteristic. Then $G$ has a subgroup of index 2 not containing $t$ (and so $G$ is not simple), except when $F \approx A_{5}$ or $F \approx$ $\operatorname{PSL}\left(2,3^{2 n+1}\right)(n \geqq 1)$.

Of course these are true exceptions, for the centralizer of an involution $t$ in the Janko simple group of order 175,560 has the form $C(t) \approx\langle t\rangle \times A_{s}$ (Janko (1966)); while in the simple Ree groups $C(t)$ has the structure $C(t) \approx$ $\langle t\rangle \times \operatorname{PSL}\left(2,3^{2 n+1}\right)(n \geqq 1)$ (Janko and Thompson (1966)). 
The notation follows that of Carter (1972) in which standard facts on the Chevalley groups may be found.

\section{Straightforward cases}

In this section Theorem 1.1 is proved when $F$ is isomorphic to one of the following simple Lie groups: $G_{2}(q), F_{4}(q), E_{6}(q), E_{8}(q),{ }^{3} D_{4}\left(q^{3}\right),{ }^{2} E_{6}\left(q^{2}\right)$ or ${ }^{2} G_{2}\left(3^{2 n+1}\right)(n \geqq 1)$. The proof of the theorem is straightforward in these cases, because when $F \approx^{2} G_{2}\left(3^{3 n+1}\right)(n \geqq 1)$ a Sylow 2-subgroup of $G$ is abelian and appeal to the theorem of Walter (1969) characterizing such groups yields the result; while in the remaining cases every involution in $F$ is a square in $F$ and the theorem follows easily from (2.1) and (2.2) below.

Proof of Theorem 1.1. First consider the case when $F \approx{ }^{2} G_{2}\left(3^{2 n+1}\right)$ ( $n \geqq 1)$, the twisted Ree group of type $G_{2}$ over the field $\mathbf{F}_{3^{2 n+1}}(n \geqq 1)$. This group has only one class of involutions and as noted above if $x$ is any involution in $F, C_{F}(x) \approx\langle x\rangle \times \operatorname{PSL}\left(2,3^{2 n+1}\right)$. Thus a Sylow 2-subgroup of $G$ is elementary abelian of order 8 , and so a Sylow 2-subgroup of $G$ is elementary abelian of order 16 .

Without loss of generality we may assume $O(G)=1$, where $O(G)$ is the maximal normal odd order subgroup in $G$. Then if $O^{\prime}(G)$ is the minimal normal subgroup of odd index in $G, C(t) \cap O^{\prime}(G) \triangleleft C(t)$ and contains a Sylow 2-subgroup of $G$. Thus $\langle t\rangle \times F \subseteq O^{\prime}(G)$. But both $G$ and $\langle t\rangle \times F$ have 2-order 16 so by a theorem of Walter (1969) characterizing groups with abelian Sylow 2-subgroups $O^{\prime}(G)=\langle t\rangle \times F$. Therefore $F \triangleleft G$ and $G / F$ has order $2 k, k$ odd. Thus by a theorem of Burnside $G / F$ has a subgroup of index 2 not containing $t F$ and the conclusion of Theorem 1.1 follows.

Now consider the remaining cases. Since $t$ is central, $C(t)$ contains a Sylow 2-subgroup of $G$ of form $S=\langle t\rangle \times M$ where $M$ is a Sylow 2-subgroup of $F$. We show $t$ is not fused (that is conjugate in $G$ ) to any involution in $M$ and use the following lemma of Thompson (1968).

Lemma 2.1. Let $M$ be a subgroup of index 2 in a Sylow 2-subgroup $S$ of a finite group $G$. Let $t$ be an involution in $S \backslash M$ which is not fused to any element of $M$. Then $G$ has a (normal) subgroup of index 2 not containing $t$.

Now the structure of $C(t)$ shows $t$ cannot be fused with an involution which is a square in $F$. More generally if $\left\langle L^{2}\right\rangle$ denotes the group generated by the squares of elements of a subgroup $L$ of $G$, the following holds:

LeMma 2.2. $t$ is not fused to any involution $x \in\left\langle C_{F}(x)^{2}\right\rangle$.

Proof. Suppose on the contrary $x=\prod_{i=1}^{m} x_{i}^{2}$ where $x_{i} \in C_{F}(x)(m$ a 
positive integer) and $t=x^{a}$, some $a \in G$. Then $t=\prod_{i=1}^{m}\left(x_{i}^{a}\right)^{2}$. But $x_{i} \in C_{F}(x) \subseteq C(x)$ so $x_{i}^{a} \in C(x)^{a}=C(t)$. Thus $t \in\left\langle C(t)^{2}\right\rangle \subseteq F$, a contradiction. But in the remaining simple Lie groups above, every involution is a square and so the conclusion of Theorem 1.1 follows immediately from (2.1) and (2.2). In the table below we list these groups, together with the corresponding Dynkin diagram, a representative of each class of involutions and the order 4 element of which it is a square.

\begin{tabular}{|c|c|c|c|}
\hline Group & $\begin{array}{c}\text { Dynkin } \\
\text { Diagram }\end{array}$ & Representatives & $\begin{array}{c}\text { Order } 4 \\
\text { elements }\end{array}$ \\
\hline$G_{2}(q)$ & 12 & $h_{1}$ & $n_{1}$ \\
\hline$F_{4}(q)$ & & $\begin{array}{l}h_{2} h_{4} \\
h_{1} h_{3}\end{array}$ & $\begin{array}{l}n_{2} n_{4} \\
n_{1} n_{3}\end{array}$ \\
\hline$E_{\varsigma}(q),{ }^{2} E_{6}\left(q^{2}\right)$ & 12356 & $\begin{array}{l}h_{2} h_{5} \\
h_{1} h_{3} h_{6}\end{array}$ & $\begin{array}{l}n_{2} n_{5} \\
n_{1} n_{3} n_{6}\end{array}$ \\
\hline$E_{8}(q)$ & 1234578 & $\begin{array}{l}h_{2} h_{4} h_{6} \\
h_{6} h_{7}\end{array}$ & $\begin{array}{l}n_{2} n_{4} n_{6} \\
n_{6} n_{7}\end{array}$ \\
\hline${ }^{3} D_{4}\left(q^{3}\right)$ & 12.4 & $h_{2}$ & $n_{2}$ \\
\hline
\end{tabular}

The representatives chosen are found from Iwahori (1970). The element $h_{i}=h_{i}(-1)$ and the element $n_{i}=x_{p_{i}}(1) x_{-p_{i}}(-1) x_{p_{i}}(1)$ is a generator of the subgroup $N$ in the Chevalley group. $n_{i}^{2}=h_{i}$, and for $i \neq j$ we have $n_{i} n_{i}=n_{j} n_{i}$ if and only if node $i$ and node $j$ are not connected in the Dynkin diagram.

\section{The case $E_{7}(q)$}

Finally consider the case when $F \approx E_{7}(q)$, the adjoint Chevalley group of type $E_{7}$ over the finite field $k=\mathbf{F}_{q}$ of $q$ elements ( $q$ odd).

Let $\Phi$ be the set of roots of a complex semi-simple Lie algebra of type $E_{7}$ relative to a Cartan subalgebra. For a fixed ordering of $\Phi$ let $\Phi^{+}$be the positive roots containing a fundamental system $\Pi=\left\{p_{1}, p_{2}, \cdots, p_{7}\right\}$, with Dynkin diagram

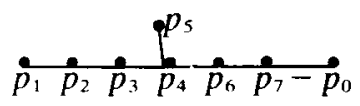

where $p_{0}$ is the highest root in $\Phi^{+}, p_{0}=p_{1}+2 p_{2}+3 p_{3}+4 p_{4}+2 p_{5}+3 p_{6}+2 p_{7}$.

Let $W=\left\langle w_{r} \mid r \in \Phi\right\rangle$ be the Weyl group of $\Phi$, and $w_{0} \in W$ be the symmetry which interchanges $p_{3}$ and $p_{6}, p_{2}$ and $p_{7}, p_{1}$ and $-p_{0}$, and fixes $p_{4}$ and $p_{5}$. 
Let $E=\langle x,(t) \mid r \in \Phi, t \in k\rangle$ be the associated adjoint Chevalley group over the field $k$, which contains the subgroup $H=\left\langle h_{r}(t) \mid r \in \Phi, t \in \dot{k}\right\rangle$, where $\dot{k}=\langle\kappa\rangle$ is the multiplicative group of $k$. The universal Chevalley group of type $E_{7}$ has centre $\left\langle h_{1}(-1) h_{3}(-1) h_{5}(-1)\right\rangle$ of order 2 , so the adjoint group $E$ is generated by the elements $x_{r}(t)(r \in \Phi, t \in k)$ satisfying the usual relations for the universal group with the additional relation $h_{1}(-1) h_{3}(-1) h_{5}(-1)=1$.

Let $K$ be the algebraic closure of $k$, and for the extension field $\mathbf{F}_{q^{2}}$ of $k$ in $K$, let $\dot{\mathbf{F}}_{q^{2}}=\langle\rho\rangle$. Put $i=\rho^{\left(q^{2}-1\right) / 4}$ and $\sqrt{\kappa}=\rho^{(q+1) / 2}$. Denote by $\bar{E}=$ $\left\langle x_{r}(t) \mid r \in \Phi, t \in K\right\rangle$ the connected linear group over $K$ containing $E$ as a subgroup in the natural way, and $\bar{H}=\left\langle h_{r}(t) \mid r \in \Phi, t \in \dot{K}\right\rangle$.

The mapping $x_{r}(t) \rightarrow x_{r}\left(t^{q}\right)(r \in \Phi, t \in K)$ on the generators of $\vec{E}$ extends to the Frobenius automorphism $\sigma$ of $\bar{E}$. For a subset $X$ of $\bar{E}$ invariant under $\sigma$, let $X_{\sigma}$ denote the fixed points of $\sigma$ in $X$. Then $E^{*}=\vec{E}_{\sigma}$ is the group of $k$ rational points in $\bar{E}$. One knows $\left|E^{*}: E\right|=2, E^{*}=E . H^{*}$ and $E \cap H^{*}=H$, where $H^{*}=\bar{H}_{\sigma}$. In fact $E^{*}=\left\langle h_{1}(\sqrt{\kappa}) h_{3}(\sqrt{\kappa}) h_{5}(\sqrt{\kappa})\right\rangle$.E.

The proof of Theorem 1.1 requires the classes of involutions and their centralizers in E. First, from the results of Iwahori (1970), we give the classes and their centralizers in $\bar{E}$.

LEMMA 3.1. (i) There are three classes of involutions in $\bar{E}$ having the following representatives in $H^{*}$ :

$$
\begin{aligned}
z & =h_{1}(-1) \\
u_{1} & =h_{1}(i) h_{2}(-1) h_{3}(-i) h_{5}(i) \\
u_{2} & =h_{1}(i) h_{3}(-i) h_{4}(-1) h_{5}(i) h_{7}(-1) .
\end{aligned}
$$

(ii) If $C^{0}$ is the connected component of the identity for the centralizer $C$ of any of the above involutions then $C / C^{0}$ is finite abelian. Coset representatives of $C^{\prime \prime}$ in $C$ are certain $n_{w} \in N=N_{E}(H), w \in W$, where the $n_{w}$ can be chosen to have the same order as $w$. Further $C_{0}=\bar{H} . X$ where $X$ is a connected semisimple algebraic group and $X \triangleleft C^{0}$. The following table lists for $z, u_{1}$ and $u_{2}$ the simple components of $X$, the corresponding set of fundamental roots, the order of

\begin{tabular}{|c|c|c|c|c|}
\hline$z$ & $A_{1}, D_{6}$ & $i \overrightarrow{346 \overrightarrow{70}}$ & 1 & 1 \\
\hline$u_{1}$ & $A_{7}$ & $\begin{array}{c}1234670 \\
59\end{array}$ & 2 & $n_{w_{0}}$ \\
\hline$u_{2}$ & $E_{6}$ & 23467 & 2 & $n_{w_{0}}$ \\
\hline
\end{tabular}
$C / C^{0}$ and the coset representatives in $N$.

Representatives Components Root Structure $\left|C / C^{0}\right| \quad n_{w}$ 
Proof. If $\Gamma_{\pi}$ is the $\mathbf{Z}$-lattice spanned by $\Pi, \vec{H}$ may be identified with $\operatorname{Hom}\left(\Gamma_{\pi}, \dot{K}\right)$. Then (ii) follows from proposition 1 in Iwahori (1970) with $X=$ $\left\langle X,(t) \mid r \in \Phi_{\chi}, t \in K\right\rangle$ where the involution $h \in \bar{H}$ corresponds to $\chi \in$ $\operatorname{Hom}\left(\Gamma_{\pi}, \dot{K}\right)$ and $\Phi_{\chi}=\{r \in \Phi \mid \chi(r)=1\}$. The involutions $z, u_{1}, u_{2}$ correspond to $\chi_{\lambda_{2}}(2), \chi_{\lambda_{5}}(2), \chi_{\lambda_{1}}(1)$ respectively in Iwahori (1970) and the classes for $\bar{E}$ (giving (i)) and the $\Phi_{\star}$ for each of these involutions are given on pages F23, F24 of that paper.

LEMMA 3.2. (i) There are 5 classes of involutions in $E^{*}$ with representatives $z, u_{i}, u_{i}^{a}(i=1,2)$ where $n_{w_{10}}=a^{\sigma} \cdot a^{-1}(a \in \bar{E})$.

(ii) There are 3 classes of involutions in $E$ with representatives:

$$
\begin{aligned}
& z, u_{i}(i=1,2) \text { when } q \equiv 1(\bmod 4) \\
& z, u_{i}^{a}(i=1,2) \text { when } q \equiv-1(\bmod 4) \text {. }
\end{aligned}
$$

Proof. By a lemma of Steinberg (10.1 in Steinberg (1968)) if $n_{w} \in \bar{E}$, $n_{w}=x^{\sigma} x^{-1}$ for some $x \in \bar{E}$. Then proposition 6 in Iwahori (1970) shows that for any involution $h \in H^{*}$ the map

$$
C_{\bar{F}}(h) / C_{\bar{E}}^{(!}(h) \rightarrow\left(h^{\bar{E}} \cap E^{*}\right) / E^{*},
$$

(the $E^{*}$ class of $h$ lying in the $\bar{E}$ class of $h$ ), defined by: $n_{w} \rightarrow\left(h^{x}\right)^{E^{*}}$ (the $E^{*}$ class of $h^{x}$ ), is a bijection. (i) now follows from (3.1) taking $n_{w_{10}}=a^{\sigma} a^{-1}$ $(a \in \bar{E})$ and (ii) is contained in Iwahori (1970) on pages F26, F27.

In order to treat the cases $q \equiv \pm 1(\bmod 4)$ uniformly we observe (for $i=1,2)$,

$$
C_{F}-\left(u_{i}^{a}\right)=C_{\bar{E}}\left(u_{i}^{a}, \sigma\right) \underset{a^{-1}}{\approx} C_{\bar{E}}\left(u_{1}, \sigma^{a^{-1}}\right)=C_{\bar{E}_{\theta}}\left(u_{i}\right)
$$

where $\bar{E}_{\theta}$ is the group of fixed points of $\theta=\sigma^{a^{-1}}=\sigma n_{w_{0}}$ in $\bar{E}$. So when $q \equiv-1$ $(\bmod 4)$ it is convenient to let $E(-1)=E^{a^{-1}}$, of index 2 in $\bar{E}_{\theta}$, and consider the centralizer of $u_{i}$ in $E(-1)$, since $C_{E}\left(u_{i}^{a}\right) \underset{a^{-1}}{\approx} C_{E(-1)}\left(u_{i}\right)$. Because $\theta=\sigma n_{\left.w_{i}\right)}$ these centralizers involve twisted Lie groups. We also put $H(-1)=H^{a^{-1}}$, $H^{*}(-1)=H^{* a^{-1}}, \quad E(1)=E, \quad H(1)=H, \quad H^{*}(1)=H^{*}$ and assume $q \equiv \varepsilon$ $(\bmod 4),(\varepsilon \pm 1)$ in the following.

LEMMA 3.3. (i) $C_{F}(z)=H . L(z)$ where $L(z)$ is a central product of Lie groups of type $A_{1}$ and $D_{h}$. Further $z$ is the only central class in $E$ and $z$ is $a$ square in $E$.

(ii). $C_{E(e)}\left(u_{1}\right)=\left\langle h_{1}\right\rangle L\left(u_{1}\right)$, where $L\left(u_{1}\right)$ is of type $A_{7}(q)(\varepsilon=1)$ or ${ }^{2} A_{7}\left(q_{2}\right)$ ( $\varepsilon=-1)$, and $h_{1} \in H(\varepsilon)$.

(iii) $C_{E(\varepsilon)}\left(u_{2}\right)=\left\langle h_{2}\right\rangle L\left(u_{2}\right)$, where $L\left(u_{2}\right)$ is of type $E_{6}(q)(\varepsilon=1)$ or ${ }^{2} E_{6}\left(q^{2}\right)$ $(\varepsilon=-1)$, and $h_{2} \in H(\varepsilon)$. 
(iv) For $i=1,2 u_{i} \in\left\langle C_{E(\varepsilon)}\left(u_{i}\right)^{2}\right\rangle$ if and only if $q \equiv \varepsilon(\bmod 8)$.

Proof. (i) From (3.1) it follows that $C_{E}(z)=H . L(z)$, where $L(z)=$ $\left\langle X_{ \pm p_{i}}(t) \mid 0 \leqq i \leqq 7, i \neq 2 ; t \in k\right\rangle$.

Let $L_{1}=\left\langle X_{ \pm p_{1}}(t) \mid t \in k\right\rangle$ and $L_{2}=\left\langle X_{ \pm p_{i}}(t) \mid i=0,3 \leqq i \leqq 7 ; t \in k\right\rangle$, then $L(z)=L_{1} \cdot L_{2}$ where $L_{1}$ is of type $A_{1}$ and $L_{2}$ is of type $D_{6}$. In fact $Z\left(L_{1}\right)=$ $\left\langle h_{1}(-1)\right\rangle$ so $L_{1} \approx \operatorname{SL}(2, q)$, and

$$
Z\left(L_{2}\right)=\left\langle h_{0}(-1) h_{5}(-1) h_{6}(-1)\right\rangle \times\left\langle h_{3}(-1) h_{5}(-1)\right\rangle=\left\langle h_{1}(-1)\right\rangle
$$

so $L_{2}$ is a homomorphic image of Spin $(12, q)$ which is not $\Omega(12, q)$. Clearly $\left[L_{1}, L_{2}\right]=1$ and $L_{1} \cap L_{2}=\left\langle h_{1}(-1)\right\rangle$ so $L(z)$ is the central product of $L_{1}$ and $L_{2}$. Further $L(z)$ is of index 2 in $C_{E}(z)$, because clearly $C_{E}(z)=\left\langle h_{2}(\kappa)\right\rangle . L(z)$ and $h_{2}(\kappa)^{2} \in L(z)$.

Also $z$ is obviously a square in $E\left(z=n_{1}^{2}\right)$ and a calculation of $\left|E: C_{E}(z)\right|$ shows $z$ is central in $E$.

(ii) For $i=1,2, C_{E(\varepsilon)}^{0}\left(u_{i}\right)=E(\varepsilon) \cap C_{E}^{0}\left(u_{i}\right)$. So from (3.1) $C_{E(\varepsilon)}^{0}\left(u_{i}\right)$ is of index 2 in $C_{E(\varepsilon)}\left(u_{i}\right)$ with coset representative $n_{w_{i}}$, and $C_{E(\varepsilon)}^{0}\left(u_{i}\right)=H(\varepsilon) . L\left(u_{i}\right)$, with $L\left(u_{i}\right) \triangleleft C_{E(e)}\left(u_{i}\right)$.

Now $L\left(u_{1}\right)=\left\langle X_{ \pm p_{i}} \mid 0 \leqq i \leqq 7, i \neq 5\right\rangle$ is of type $A_{7}(q)(\varepsilon=1)$ or of type ${ }^{2} A_{7}\left(q^{2}\right)(\varepsilon=-1)$. If $d=(q-\varepsilon, 8)$ and $\gamma=\rho^{\left(q^{2}-1\right) / a}$ then

$$
\begin{aligned}
Z\left(L\left(u_{1}\right)\right) & =\left\langle h_{1}(\gamma) h_{2}\left(\gamma^{2}\right) h_{3}\left(\gamma^{3}\right) h_{4}\left(\gamma^{4}\right) h_{6}\left(\gamma^{5}\right) h_{7}\left(\gamma^{6}\right) h_{0}\left(\gamma^{7}\right)\right\rangle \\
& =\left\langle h_{1}\left(\gamma^{2}\right) h_{2}\left(\gamma^{4}\right) h_{3}\left(\gamma^{6}\right) h_{5}\left(\gamma^{2}\right)\right\rangle .
\end{aligned}
$$

Thus when $q \equiv \varepsilon(\bmod 8), Z\left(L\left(u_{1}\right)\right)=\left\langle u_{1}\right\rangle$, and when $q \equiv 4+\varepsilon(\bmod 8)$, $Z\left(L\left(u_{1}\right)\right)=1$. So in the latter case $L\left(u_{1}\right) \approx \operatorname{PSL}(8, q)(\varepsilon=1)$ or $L\left(u_{1}\right) \approx$ $\operatorname{PSU}(8, q)(\varepsilon=-1)$.

In fact if $\lambda=\rho^{q-\varepsilon}$ (so $\lambda^{q}=\lambda=\kappa(\varepsilon=1)$ and $\lambda^{q}=\lambda^{-1}(\varepsilon=-1)$ ) it is easily seen that

$$
H(\varepsilon) \cdot L\left(u_{1}\right)=\left\langle h_{1}\right\rangle \cdot L\left(u_{1}\right)
$$

where $h_{1}=h_{1}(\lambda) h_{2}\left(\lambda^{2}\right) h_{3}\left(\lambda^{3}\right) h_{4}\left(-\lambda^{2}\right) h_{5}(\lambda)$.

Note: if $\sqrt{\lambda}=\rho^{(q-\varepsilon) / 2}$, and $g_{1}=h_{1}(\sqrt{\lambda}) h_{2}(\lambda) h_{3}(\sqrt[3]{\lambda}) h_{4}(-\lambda) h_{5}(\sqrt{\lambda})$ then $g_{1} \in$ $H^{*}(\varepsilon)-H(\varepsilon)$ and $g_{1}^{2}=h_{1}$. Since $h_{1}{ }^{2} \in L\left(u_{1}\right)$ and $h_{1}^{n_{\omega_{0}}}=h_{1}^{-1} \equiv h_{1}\left(\bmod L\left(u_{1}\right)\right)$, $C_{E(r)}\left(u_{1}\right) / L\left(u_{1}\right) \approx Z_{2} \times Z_{2}$, the 4-group.

Hence $L\left(u_{1}\right)=L\left(u_{1}\right)^{\prime} \subseteq C_{E(f)}\left(u_{1}\right) \subseteq\left\langle C_{E(f)}\left(u_{1}\right)^{2}\right\rangle \subseteq L\left(u_{1}\right)$.

(iii) $L\left(u_{2}\right)=\left\langle X_{ \pm p_{1}} \mid 2 \leqq i \leqq 7\right\rangle$ is of type $E_{6}(q)(\varepsilon=1)$ or of type ${ }^{2} E_{6}\left(q^{2}\right)$ $\varepsilon=-1)$, with a centre of order $(3, q-\varepsilon)$. In fact

$$
H(\varepsilon) . L\left(u_{2}\right)=\left\langle h_{2}\right\rangle . L\left(u_{2}\right)
$$

where $h_{2}=h_{1}(\lambda) h_{3}\left(\lambda^{3}\right) h_{4}\left(\lambda^{2}\right) h_{5}(\lambda) h_{7}\left(\lambda^{2}\right)$. Since $h_{2}^{(q-e) / 2}=1$ and $h_{2}^{n_{w_{0}}}=h_{2}^{-1}$, $C_{E(\varepsilon)}\left(u_{2}\right) / L\left(u_{2}\right)$ is dihedral. 
Thus $\left\langle C_{E(\varepsilon)}\left(u_{2}\right)^{2}\right\rangle=C_{E(\varepsilon)}\left(u_{2}\right)^{\prime}$ is of index 2 in $H(\varepsilon) L\left(u_{2}\right)$.

(iv) Since $h_{i}^{(q-\varepsilon) / 4}=u_{i}, u_{i} \in\left\langle C_{E(\varepsilon)}\left(u_{i}\right)^{2}\right\rangle$ if and only if $q \equiv \varepsilon(\bmod 8)$, and in fact when $q \equiv \varepsilon(\bmod 8), u_{i}=\left(h_{i}^{(q-\varepsilon) / 8}\right)^{2}$ is actually a square in $E$.

A calculation of $\left|E(\varepsilon): C_{E(\varepsilon)}\left(u_{i}\right)\right|$ shows the $u_{i}$ are not central $(i=1,2)$ in $E(\varepsilon)$.

We now give the proof of (1.1) when $F \approx E_{7}(q)$.

Proof of (1.1). We put $F=E(\varepsilon)$ where as above $q \equiv \varepsilon(\bmod 4), \varepsilon=$ \pm 1 ; and relabel $z^{a}$, the representative of the central class in $E(-1), z$. Thus the classes in $F$ have representatives $z, u_{1}$ and $u_{2}$. We show $t$ is not fused to any of these three involutions and the theorem then follows from (2.2). By (3.3) when $q \equiv \varepsilon(\bmod 8) z, u_{1}$ and $u_{2}$ are all squares in $F$ and the conclusion of the theorem follows from (2.1).

Thus assume $q \equiv 4+\varepsilon(\bmod 8)$. A maximal set of representatives of the classes of involutions in $C(t)=\langle t\rangle \times F$ is the set $\left\{t, z, u_{i}, t z, t u_{i} \mid i=1,2\right\}$.

(a) Again $z$ is a square in $F$ by (3.3) so $t \neq z$ by (2.2).

(b) Suppose $t z \sim t$ in $G$, say $(t z)^{b}=t$ some $b \in G$. As $t \in C(t z), t^{b} \in C(t)$ and is thus conjugate to one of the involutions above. Suppose $t^{b} \sim u_{i}$ or $t u_{i}$ $(i=1,2)$. In fact we may assume $t^{b}=u_{i}$ or $t u_{i}$. In either case

$$
\begin{gathered}
C(t, z)^{b}=C(t ; t z)^{b}=C\left(t, u_{i}\right), \\
\text { hence }\left(C(t, z)^{(\infty)}\right)^{b}=C\left(y, u_{i}\right)^{(\infty)},
\end{gathered}
$$

where for a subgroup $L$ of $G, L^{(x)}$ is the last term in the derived series of $L$.

Now $C(t, z)=\langle t\rangle \times C_{F}(z)$, so $C(t, z)^{(x)}=C_{F}(z)^{(\infty)}=L(z)$ and similarly $C\left(t, u_{i}\right)^{(x)}=L\left(u_{i}\right)$. This gives a contradiction since by $(3.3) L(z)$ and $L\left(u_{i}\right)$ are not isomorphic. Thus $t^{b} \sim t z$ and again we may assume $t^{b}=t z$ so $t z \rightarrow t \rightarrow t z$ under $b$. Therefore $\langle C(t, z), b\rangle$ is a subgroup of order $2|C(t, z)|$, contradicting the fact that $C(t, z)$ contains a Sylow 2-subgroup of $G$ (since $z$ is central in $F$ ). Thus $t z \not t$ in $G$.

(c) Suppose $u_{i}$ is conjugate to $t$ in $G$ so $u_{i}^{c_{i}}=t$, some $c_{i} \in G$. Now $u_{1}^{n_{s}}=$ $u_{1} h_{5}(-1)$ and $u_{2}^{n_{0} n_{1}}=u_{2} h_{0}(-1) h_{1}(-1)=u_{2} h_{3}(-1) h_{6}(-1)$, where $\cdot n_{5}, n_{0} n_{1}$, $z_{1}=h_{5}(-1), z_{2}=h_{3}(-1) h_{6}(-1) \in F$. Thus $u_{i} \sim u_{i} z_{i}$ where $z_{i}$ is central in $F$ (since $z_{i}$ is clearly a square in $F$ for $i=1,2$ ). Conjugating this relation by $c_{i}$, and assuming for the moment $z_{i}^{c_{i}}=z_{i}$, we have $t \sim t z_{i}$ in $G$. However $t z_{i} \sim t z$ in $G$ and this contradicts the result of (b).

To justify the assumption, recall we are assuming $u_{i}^{c_{i}}=t$. Then as in (b), since $L(z), L\left(u_{1}\right)$ and $L\left(u_{2}\right)$ are not isomorphic by (3.3), we may suppose $t^{c_{i}}=$ $u_{i}$ or $t u_{i}$. In either case $c_{i}$ centralizes $C\left(t, u_{i}\right)$ and so induces an automorphism on $C\left(t, u_{i}\right)^{(\infty)}=L\left(u_{i}\right)$. But $z_{i} \in L\left(u_{i}\right)$ and by a theorem of Steinberg (1968) every automorphism $\psi$ of a Chevalley group is of form $\psi=f g d i$ where $f$ is a 
field, $g$, a graph, $d$ a diagonal and $i$ an inner automorphism. As $z_{i}$ is fixed by field, graph and diagonal automorphisms $z_{i}^{c_{i}}=z_{i}^{f_{i}}$ some $f_{i} \in L\left(u_{i}\right) \subseteq F$. Replacing $c_{i}$ by $c_{i}^{\prime}=c_{i} f_{i}^{-1}$ we have $u_{i}^{c^{i}}=t$ and $z_{i}^{c_{i}}=z_{i}$ as assumed.

Therefore $t$ is not fused to any involution in $F$ and the conclusion of the theorem follows from (2.1). This completes the proof of (1.1).

\section{References}

Roger W. Carter (1972), Simple Groups of Lie Type (Pure and Appl. Math., 28. Interscience [John Wiley \& Sons], New York, London, Sydney, 1972).

M. J. Curran, 'Groups with decomposable involution centralizers', submitted for publication. Nagayoshi Iwahori (1970), 'Centralizers of involutions in finite Chevalley groups', Seminar on Algebraic Groups and Related Finite Groups, F1-F29 (Lecture Notes in Mathematics, 131. Springer-Verlag, Berlin, Heidelberg, New York, 1970).

Zvonimir Janko (1966), 'A new finite simple group with abelian Sylow 2-subgroups and its characterization', J. Algebra 3, 147-186.

Zvonimir Janko and John G. Thompson (1966), 'On a class of finite simple groups of Ree', $J$. Algebra 4, 274-292.

Robert Steinberg (1968), Endomorphisms of Linear Algebraic Groups (Memoirs Amer. Math. Soc. 80. Amer. Math. Soc., Providence, Rhode Island, 1968).

John G. Thompson (1968), 'Nonsolvable finite groups all of whose local subgroups are solvable', Bull. Amer. Math. Soc. 74, 383-437.

John H. Walter (1969), 'The characterization of finite groups with abelian Sylow 2-subgroups', Ann. of Math. (2) 89, 405-514.

Hiroyoshi Yamaki (1972), 'On Janko's simple group of order 175560', Osaka J. Math. 9, 111-112.

Department of Mathematics,

University of Auckland,

New Zealand.

Present address:

Department of Mathematics,

University of Otago,

New Zealand. 